\title{
An alternative to killing? Treatment of reservoir hosts to control a vector and pathogen in a susceptible species
}

\author{
R. PORTER ${ }^{1,2} \uparrow$, R. A. NORMAN ${ }^{2}$ and L. GILBERT ${ }^{1 *}$ \\ ${ }^{1}$ The Fames Hutton Institute, Macaulay Drive, Craigiebuckler, Aberdeen AB15 8QH, UK \\ ${ }^{2}$ Computing Science and Mathematics, University of Stirling, Stirling FK9 4LA, UK
}

(Received 7 March 2012; revised 6 Fuly, 9 fuly and 16 fuly 2012; accepted 23 fuly 2012; first published online 3 September 2012)

\section{SUMMARY}

Parasite-mediated apparent competition occurs when one species affects another through the action of a shared parasite. One way of controlling the parasite in the more susceptible host is to manage the reservoir host. Culling can cause issues in terms of ethics and biodiversity impacts, therefore we ask: can treating, as compared to culling, a wildlife host protect a target species from the shared parasite? We used Susceptible Infected Recovered (SIR) models parameterized for the tick-borne louping ill virus (LIV) system. Deer are the key hosts of the vector (Ixodes ricinus) that transmits LIV to red grouse Lagopus lagopus scoticus, causing high mortality. The model was run under scenarios of varying acaricide efficacy and deer densities. The model predicted that treating deer can increase grouse density through controlling ticks and LIV, if acaricide efficacies are high and deer densities low. Comparing deer treated with $70 \%$ acaricide efficacy with a $70 \%$ cull rate suggested that treatment may be more effective than culling if initial deer densities are high. Our results will help inform tick control policies, optimize the targeting of control methods and identify conditions where host management is most likely to succeed. Our approach is applicable to other host-vector-pathogen systems.

Key words: acaricide, apparent competition, culling, deer, grouse, louping ill, management, mathematical modelling.

\section{INTRODUCTION}

In complex systems where multiple animal or plant species interact with pathogens or their vectors it can be difficult to devise effective disease management strategies. Vector-borne pathogens, in particular, frequently affect multiple species, especially where the vector is a generalist parasite. For example, mosquitoes can transmit West Nile virus between birds, humans and horses (Kulasekera, 2001). Where multiple host species share a common vector or pathogen, parasite-mediated apparent competition can occur such that changes in the population of the less susceptible reservoir host can affect the population of the more susceptible host (Holt, 1977). For example, eastern grey squirrels Sciurus carolinensis are reservoir hosts for squirrel poxvirus and appear not to suffer clinical symptoms, but red squirrels Sciurus vulgaris suffer high mortality when infected (Tompkins et al. 2002). Partly through poxvirusmediated apparent competition the spread of grey squirrels in the United Kingdom has resulted in the decimation of many British red squirrel populations

* Corresponding author: The James Hutton Institute, Macaulay Drive, Craigiebuckler, Aberdeen AB15 8QH, UK. Tel: + 44 (0)1224 395187. Fax: + 44 (0) 8449285429. Lucy.Gilbert@hutton.ac.uk

$\uparrow$ Current address: Department of Engineering and Maths, Sheffield Hallam University, City Campus, Howard Street, Sheffield S1 1WB, UK.
(Rushton et al. 2000). Therefore, methods to protect surviving red squirrel populations often involve culling grey squirrels. Culling wildlife hosts can, however, cause serious issues in terms of ethics, welfare and public opinion, as well as having biodiversity impacts, and therefore more benign alternative methods may be preferable.

Here we use a mathematical model to predict the effectiveness of treating versus culling a wildlife host species to benefit a susceptible species by protecting it from a shared parasite. We test this using a tick-borne pathogen system-louping ill virus (LIV) - because the incidence of tick-borne diseases is increasing in many parts of Europe (e.g. Randolph, 2004), impacting on human and animal health, and finding effective control methods is an increasingly urgent issue. Ticks Ixodes ricinus are the most important vector in Europe of many human and animal pathogens (e.g. Borrelia burgdorferi sensu lato, the agent of Lyme borreliosis and the tick-borne encephalitis (TBE) complex of viruses). LIV is one of the TBE viruses and is of increasing concern in the UK as it kills sheep Ovis aries and red grouse Lagopus lagopus scoticus, an economically valuable game bird. I. ricinus tick burdens on red grouse are increasing (Kirby et al. 2004) while red grouse populations have generally declined in many areas. This has led to various largescale management strategies in attempts to reduce ticks with the aim of increasing red grouse densities for hunting purposes. 
Management of hosts to control ticks and tick-borne diseases

Ixodes ricinus has 3 active life stages (larvae, nymphs and adults) that each require a blood meal from each of 3 different hosts. Larvae tend to feed on small hosts such as small mammals and birds but, because transovarial transmission is absent or rare, larvae do not carry LIV (Gaunt, 1997). Nymphs feed on a wide range of host types, including birds, mountain hares Lepus timidus and roe deer Capreolus capreolus (e.g. Kiffner et al. 2011) and may be infected with LIV. The prevalence of LIV is likely to vary depending on the densities of transmission hosts (e.g. Gilbert et al. 2001) but is generally thought to be low, for example, Gaunt, 1997 found that 0.3\% of nymphs were infected. Adult I. ricinus tend to feed on larger hosts such as mountain hares, sheep and deer; indeed, deer are the most important $I$. ricinus tick reproduction hosts in many parts of Europe and North America (e.g. Gray, 1998). While red grouse, mountain hares and sheep can transmit LIV to biting $I$. ricinus ticks, deer however, cannot transmit LIV to ticks and appear unaffected by the virus (Jones et al. 1997), in contrast to red grouse that have been shown in laboratory studies to suffer $78 \%$ mortality in experimentally infected birds (Reid, 1975). LIV is a particularly interesting system because it can cause a special type of apparent competition (Gilbert et al. 2001), whereby tick hosts such as deer may affect grouse populations indirectly, through multiplication of the vector rather than the pathogen itself. From this theory, therefore, if we make the assumption that red grouse abundance is adversely impacted by LIV infection, we can predict that managing tick hosts in a way that controls tick abundance may improve the abundance of red grouse that use the same habitat. What management methods can be used to control tick hosts? Using fencing to exclude deer can result in fewer ticks (e.g. Ruiz-Fons and Gilbert, 2010) and there are general associations between deer abundance and questing tick abundance (e.g. Stafford et al. 2003; Ruiz-Fons and Gilbert, 2010), implying that excluding or culling deer may be a useful tool for controlling ticks and, therefore, perhaps also tickborne pathogens. However, severe reductions in densities of ecosystem engineers such as roe and red deer Cervus elephus can have cascading impacts on the environment, and the cultural and economic value of that species.

The treatment of livestock with acaricides is standard practice to protect the treated individual from ectoparasites and disease. Treating sheep with acaricides to reduce ticks in the environment (and vaccinating sheep against LIV), and therefore protect red grouse from LIV, has been found to be effective in an area of northern England that lacks the main alternative tick hosts, namely red deer and mountain hares (Newborn and Baines, 2012). Mathematical models have been used to explore the effectiveness of this method in areas that do have deer: the treatment of livestock was predicted to improve grouse populations only if there are very few deer (Porter et al. 2011), which clearly has implications for areas with large populations of the main alternative tick hosts, such as Scotland. Therefore, an alternative to treating sheep or culling deer may be to treat deer with acaricide for the purposes of managing ticks, tickborne diseases and target species abundance. Studies in the USA, in which white tailed deer Odocoileus virginianus acquired acaricide from rollers at feeding stations, have found that this method can reduce the density of $I$. scapularis nymphs in some experimental areas by at least 70\% (Brei et al. 2009).

Our objective was to apply the idea of treating deer with acaricide to the LIV system, by using mathematical models to predict the effectiveness of treating, as compared to killing, deer as a management strategy to increase red grouse abundance by controlling ticks and LIV. In doing so we are testing the general concept of treating wildlife hosts in order to reduce vectors and disease and improve target species abundance, as well as comparing treatment with culling. In addition, we are exploring the transferability of a method that can work in one situation (acaricide treatment of white-tailed deer to control I. scapularis and Lyme disease in the USA) to another related situation (acaricide treatment of roe and red deer to control I. ricinus and LIV in the UK).

Mathematical modelling allows us to address questions that can be difficult to test empirically. In particular, we use the model to answer the following specific questions, aimed at informing practical management of our test system. (i) Can acaricidetreated deer increase red grouse abundance by reducing $I$. ricinus tick abundance? (ii) What acaricide efficacy is needed to control ticks and increase grouse abundance? (iii) How does acaricide treatment compare to culling deer? (iv) Could combining acaricide treatment with deer culling be the most effective management strategy for increasing grouse abundance?

\section{MATERIALS AND METHODS}

\section{The model}

To predict the effectiveness of managing deer to control ticks and LIV we used a susceptible-infectiousrecovered (SIR) type model of coupled differential equations (similar to that described by Gilbert et al. 2001) and included the effect of deer management on the tick population, LIV prevalence and red grouse population. The model equations are:

$$
\begin{aligned}
\frac{d G_{s}}{d t} & =\left(a_{g}-s_{g} G\right) G-b_{g} G_{s}-\beta_{1} T_{i} G_{s} \\
\frac{d G_{i}}{d t} & =\beta_{1} T_{i} G_{s}-\left(\alpha+b_{g}+\gamma\right) G_{i}
\end{aligned}
$$




$$
\begin{aligned}
\frac{d G_{z}}{d t}= & \gamma G_{i}-b_{g} G_{z} \\
\frac{d T_{s}}{d t}= & \left(a_{t}-s_{t} T\right) T\left((1-c)(1-d) \beta_{5} D\right)-b_{t} T_{s} \\
& -\beta_{2} T_{s} G_{i}-(1-c)\left(d \beta_{4}+\beta_{5}\right) D T_{s} \\
\frac{d T_{i}}{d t}= & \beta_{2} T_{s} G_{i}-b_{t} T_{i}-(1-c)\left(d \beta_{4}+\beta_{5}\right) D T_{i}
\end{aligned}
$$

The model grouse population, $G$, is split into 3 disease classes, susceptible $G_{s}$, infected $G_{i}$ or immune $G_{z}$. The model assumes that a susceptible grouse $G_{s}$ becomes an LIV-infected grouse $G_{i}$ when bitten by an infected tick and then has a $78 \%$ chance of dying of infection (Reid, 1975), but if it survives it becomes an immune grouse $G_{z}$. The model assumes no such acquired immunity to ticks, as there are no published data to suggest this. The model tick population, $T$, is either susceptible $T_{s}$ or infected $T_{i}$. In previous models (e.g. Gilbert et al. 2001; Laurenson et al. 2003) only the attachment of adult ticks to deer had been considered as deer do not transmit the virus and the only role deer played was to facilitate tick reproduction. Here, however, we must consider the attachment of immature ticks as they will also be affected by acaricide treatment of deer. The rates at which immature and adult ticks attach to deer $(D)$ are given by $\beta_{4}$ and $\beta_{5}$ respectively. The efficacy of the acaricide, $d$, is modelled as the proportion of ticks attaching to treated deer that are killed and each tick stage is assumed to be equally affected by the acaricide. The proportion of adult ticks that can reproduce is, therefore, $1-d$. The acaricide efficacy level was assumed to be for the treated deer population as a whole, e.g. an efficacy of $50 \%$ could be the result of $50 \%$ of the deer having all of their ticks killed or all of the deer having $50 \%$ of their ticks killed, or any appropriate combination. All adult ticks are assumed to die either as a result of acaricide use or at the natural death rate or because they have successfully laid eggs. Immature ticks are assumed to die as a result of acaricide use or natural death (including overwintering mortality). In the model the ticks killed by acaricide on deer are removed from the entire model tick population, resulting in a tick population reduction, which translates into fewer ticks available in the environment to bite grouse. This assumes homogeneous mixing spatially between deer, grouse and ticks and no host preferences in ticks.

The initial deer population may be culled by a proportion $c$ of the initial population so that the proportion of deer remaining is $1-c$. The Gilbert et al. (2001) model included mountain hares since they can host all stages of $I$. ricinus tick and transmit LIV non-viraemically (Jones et al. 1997). However, here we do not include mountain hares because most

\begin{tabular}{|c|c|c|}
\hline Parameter & $\begin{array}{l}\text { Value } \\
\text { (per month) }\end{array}$ & Definition and rationale \\
\hline$a_{g}$ & $0 \cdot 167$ & $\begin{array}{l}\text { Natural birth rate of grouse } \\
\text { (Hudson, 1992) }\end{array}$ \\
\hline$s_{g}$ & $0 \cdot 0003$ & $\begin{array}{l}\text { Density dependence constraint } \\
\text { on grouse (Porter et al. 2011) }\end{array}$ \\
\hline$b_{g}$ & $0 \cdot 087$ & $\begin{array}{l}\text { Natural death rate of grouse } \\
\text { (Hudson 1992) }\end{array}$ \\
\hline$\alpha$ & 5 & $\begin{array}{l}\text { Disease induced death rate of } \\
\text { grouse (Reid, 1975) }\end{array}$ \\
\hline$\gamma$ & $1 \cdot 25$ & $\begin{array}{l}\text { Recovery rate of infected grouse } \\
\text { (Reid, 1975) }\end{array}$ \\
\hline$a_{t}$ & $83 \cdot 33$ & $\begin{array}{l}\text { Natural birth rate of ticks } \\
\text { (Gilbert et al. 2001) }\end{array}$ \\
\hline$s_{t}$ & $0 \cdot 000002$ & $\begin{array}{l}\text { Density dependence constraint } \\
\text { on ticks (Porter } \text { et al. 2011) }\end{array}$ \\
\hline$b_{t}$ & $0 \cdot 083$ & $\begin{array}{l}\text { Natural death rate of ticks } \\
\text { (Gilbert et al. 2001) }\end{array}$ \\
\hline$\beta_{1}$ & $0 \cdot 00002$ & $\begin{array}{l}\text { The rate at which a tick bites } \\
\text { and infects a grouse (Porter } \\
\text { et al. 2011) }\end{array}$ \\
\hline$\beta_{2}$ & $9 \cdot 75 \beta_{1}$ & $\begin{array}{l}\text { The rate a tick bites a grouse } \\
\text { and becomes infected (Gilbert } \\
\text { et al. 2001) }\end{array}$ \\
\hline$\beta_{4}$ & $1 \cdot 84 \beta_{1}$ & $\begin{array}{l}\text { The rate an immature tick bites } \\
\text { a deer (Gilbert et al. 2001) }\end{array}$ \\
\hline$\beta_{5}$ & $8 \cdot 82 \beta_{1}$ & $\begin{array}{l}\text { The rate an adult female tick } \\
\text { bites a deer and reproduces } \\
\text { (Gilbert et al. 2001) }\end{array}$ \\
\hline$d$ & varied & $\begin{array}{l}\text { The efficacy of acaricide. Varied } \\
\text { for comparison. }\end{array}$ \\
\hline$c$ & varied & $\begin{array}{l}\text { The culling rate of deer. Varied } \\
\text { for comparison }\end{array}$ \\
\hline
\end{tabular}
areas investing heavily in tick control methods to improve grouse hunting are likely to be maintaining
Table 1. Description of the model parameters

hare numbers at low levels (see Laurenson et al. 2003). Model parameter values are given in Table 1 and more detailed justifications are given by Gilbert et al. (2001). It is important to test which parameters have a disproportionately large influence on model output, because variations or inaccuracies in estimates of the values of those parameters will have a large influence on the results and interpretation. The sensitivity analyses for this model are in the Online Supplementary Material.

The model was run using Mathematica Version 7.

\section{The reproductive rate of the virus, $\mathrm{R}_{0}$}

The reproductive number of a virus, $R_{0}$ is the number of new infected individuals caused by the introduction of 1 infected individual into a susceptible population. $R_{0}$ can be used to predict whether a virus is able to persist or become established in a population. If $R_{0}>1$ the virus can persist or become established, if $R_{0}<1$ the virus will die out or not become established. For tick-borne infections a state of endemic infection may be started in a wholly susceptible population by the addition of either an infected tick or an infected host; $R_{0}$ combines the number of infected ticks created by an infectious host 
and the number of infected hosts created by an infected tick. Considering the equations we can see that 1 infected grouse lives for $\left(\alpha+b_{g}+\gamma\right)^{-1}$ units of time and infects $\beta_{2} K_{t}\left(\alpha+b_{g}+\gamma\right)^{-1}$ ticks; similarly an infected tick lives for $\left((1-c)\left(d \beta_{4}+\beta_{5}\right) D+b_{t}\right)^{-1}$ units of time and infects $\beta_{1} K_{g}\left((1-c)\left(d \beta_{4}+\beta_{5}\right) D+b_{t}\right)^{-1}$ grouse. Combining these gives:

$$
R_{0}=\frac{\beta_{1} \beta_{2} K_{g} K_{t}}{\left(\alpha+b_{g}+\gamma\right)\left((1-c)\left(d \beta_{4}+\beta_{5}\right) D+b_{t}\right)}
$$

where $K_{g}$ and $K_{t}$ are the carrying capacity of grouse and ticks respectively and are given by:

$K_{g}=\frac{\alpha_{g}-b_{g}}{s_{g}}$

and

$K_{t}=\frac{\alpha_{t}(1-c)(1-d) \beta_{5} D-\left((1-c)\left(d \beta_{4}+\beta_{5}\right) D+b_{t}\right)}{s_{t}(1-c)(1-d) \beta_{5} D}$

The same formula is derived if we use equilibrium stability analysis (Anderson and May, 1981) or next generation matrices (Hartemink et al. 2008). We used the pathogen persistence threshold of $R_{0}>1$ in model outputs to determine whether LIV would invade or persist under different scenarios of deer treatment for different densities.

\section{Time series simulations}

To compare the culling of deer with the treatment of deer in a system with LIV present, we first ran the model without acaricide treatment for 100 years at a given deer density to get estimates of the grouse and tick populations when at disease-induced equilibrium. We then ran the model including acaricide treatment using the predicted pre-treatment equilibrium densities of grouse and ticks as the initial conditions. This enabled the model outputs to represent the effect of deer management scenarios on grouse and tick populations over time, starting from the time at which management (treatment or culling) began.

Our model did not incorporate the seasonality of ticks and grouse. Although populations of grouse and ticks do change with season within each year, we have previously found that incorporating seasonality into similar SIR models of the LIV-tick-grouse system does not significantly change the patterns of the outputs (Porter, 2011). However, in practice, seasonal changes in deer movements and feeding habitats will affect the likelihood of deer using acaricide application devices, and we therefore discuss this in terms of practical management.

\section{Model scenarios to answer the specific questions}

We ran the model under the following scenarios to answer each of the specific questions outlined in the
Introduction. (i) To predict the effect of treating versus not treating deer on the grouse and tick populations we ran the model with different deer densities for scenarios with and without acaricide treatment. Deer densities were varied between 5 and $25 \mathrm{~km}^{-2}$, which encompasses the range typically found for red deer in Scotland, and we fixed the herd-level acaricide efficacy at $70 \%$. It is not specified in the model how this herd-level efficacy is achieved, i.e. whether the acaricide kills $70 \%$ of ticks on $100 \%$ deer, or $100 \%$ of ticks on $70 \%$ of deer, or any appropriate combination. We chose $70 \%$ efficacy because, during trials using an acaricide applicator for wild white-tailed deer in the USA, the proportion of deer using the devices was, on average, $76 \%$ while acaricide efficacies on treated white-tailed deer of 92-97\% using 2\% amitraz (Pound et al. 2000) and $100 \%$ using $10 \%$ permethrin (Solberg et al. 2003) have been achieved. These figures produce a herdlevel efficacy of $70-76 \%$. Furthermore, tick burdens on deer from areas containing acaricide applicators averaged $3 \cdot 4$ while deer from untreated areas had $10 \cdot 8$ ticks (Sonenshine et al. 1996), equivalent to a herdlevel efficacy of $68 \cdot 5 \%$.

(ii) Since $70 \%$ acaricide efficacy might not be achievable in all areas, we ran the model under scenarios of varying acaricide efficacies and deer densities. Using the virus persistence threshold of $R_{0}=1$ we were able to predict, for areas with different deer densities, what acaricide efficacy would be needed for the grouse population to reach a density that allows economically viable grouse hunting. Commercial red grouse hunting has often been thought to need a grouse density of more than $60 \mathrm{~km}^{-2}$ to be economically viable (Hudson, 1992) and we assumed a maximum carrying capacity of grouse of $240 \mathrm{~km}^{-2}$ (L. Gilbert unpublished data; public communications Game and Wildlife Conservation Trust. Red grouse counts in 2008. Accessed 4 Jan 2012. http://www. gwct.org.uk/research__surveys/species_research/ birds/red_grouse_bap_species/1519.asp). (iii) To compare the effects of acaricide treatment with culling deer on grouse and tick densities we ran the model with an initial cull of $70 \%$ (i.e. reducing the deer density by $70 \%$ ) versus an acaricide efficacy of $70 \%$. This was done for deer densities of 10 and $20 \mathrm{~km}^{-2}$ since these densities are fairly typical for areas of Scotland managed for both deer and grouse hunting (J. Irvine unpublished data). In practice land managers tend to cull deer to a given density rather than cull a particular proportion of the population. However, by looking at culling a proportion we were able to compare culling and acaricide directly for the purposes of a direct comparison between the two management methods. (iv) Since deer populations are routinely culled for sporting or habitat related objectives in Scotland we explored the effectiveness of combining culling with treatment (first culling to reduce deer densities before, then using acaricide) by 
considering the model outputs from the previous scenarios.

For all these scenarios the model assumes that LIV causes mortality in wild red grouse at the same rate (78\% of infected grouse) as shown in laboratory experiments (Reid, 1975). Connected with this, our interpretations of the model outputs assume that mortality of grouse from LIV impacts on the grouse population more than other 'natural' causes of death. In reality, there will be great variation in the natural death rate of grouse in both space and time, depending on other factors such as habitat quality, predators and weather. A sensitivity analysis of the grouse death rate parameter, and all other model parameters, is in the Online Supplementary Material.

\section{RESULTS}

The sensitivity analysis (see Online Supplementary Material) indicated that model outputs were most sensitive to the tick parameters (tick birth rate, tick death rate and the biting rate of ticks on deer, $\beta_{5}$ ). We therefore tested our main results against variation in these parameters by varying their values by $10 \%$ in each direction. The results for varying these 3 parameters were all very similar; therefore, as an example, we show the results for the effect of varying values of the tick biting rate $\left(\beta_{5}\right)$ only on the relationship between deer density and acaricide efficacy (Fig. 1). It can be seen that the qualitative behaviour of the model was not altered by this change in the parameter and, in addition, the quantitative results do not change greatly. Nonetheless, variations in these parameters due to natural variation between areas and depending on environmental conditions will result in slight quantitative differences in model outputs, so although all the results below are termed quantitatively, they must be interpreted with caution.

\section{Can acaricide-treated deer increase grouse abundance by reducing tick density?}

The model predictions of the total grouse and tick densities over time when deer are not treated compared to when they have been treated with acaricide of efficacy $70 \%$ are shown in Fig. 2. In the presence of untreated deer the grouse population is predicted to be substantially larger when deer densities are only $5 \mathrm{~km}^{-2}$ compared with higher deer densities, and this is due to increased deer causing a predicted increase in ticks, the vectors of LIV. In contrast, however, if the deer are treated with acaricide at $70 \%$ efficacy, the model predicts that the grouse population will increase as tick densities are reduced by the acaricide for all deer densities investigated $\left(5-25 \mathrm{~km}^{-2}\right)$. If the deer density is $5 \mathrm{~km}^{-2}$ or less then the tick population (and therefore also LIV) is unable to persist and the grouse remain at carrying capacity $\left(240 \mathrm{~km}^{-2}\right)$. If the deer density is $25 \mathrm{~km}^{-2}$ or above then the tick population is so large that treatment of deer (at the 70\% efficacy assumed in this model scenario) cannot reduce the tick population sufficiently to eradicate the virus, although the grouse are still predicted to persist at a higher density than in the scenario without acaricide treatment.

\section{What acaricide efficacy is needed to control ticks and increase grouse abundance?}

Figure 3 shows the model predictions for the deer densities and efficacy levels for which grouse are predicted to (i) reach carrying capacity $\left(240 \mathrm{~km}^{-2}\right)$, (ii) reach the minimum density required for commercial hunting $\left(60 \mathrm{~km}^{-2}\right)$ and (iii) fail to reach the minimum density required for commercial hunting $\left(<60 \mathrm{~km}^{-2}\right)$ when equilibrium is obtained. The model predicted that the grouse can reach carrying capacity for deer densities of $5 \cdot 7 \mathrm{~km}^{-2}$ and below irrespective of acaricide efficacy (i.e. even with no treatment of deer). This reinforces the time series predictions (from Fig. 2a). Where deer densities rise above $6 \mathrm{~km}^{-2}$ the grouse density is reduced below the minimum for commercial hunting $\left(60 \mathrm{~km}^{-2}\right)$ if there is no acaricide treatment of deer or very low acaricide efficacies. However, increasing the acaricide efficacy means that grouse densities high enough for commercial hunting can be achieved. Indeed, if it is practically possible to apply acaricide to the deer at an efficacy of at least $73 \%$ the grouse were predicted to reach carrying capacity for all biologically reasonable deer densities (Fig. 3).

The results presented in Fig. 3 suggest that the predicted grouse density category is very sensitive to deer density such that a slight increase in deer density is predicted to cause a sudden fall in the grouse density as the $R_{0}=1$ virus persistence threshold is crossed, and this is especially true at low deer densities and low acaricide efficacies. This is because the disease is predicted to have such a large impact on the grouse population due to high disease-induced mortality (78\% in experimentally infected birds in the laboratory: Reid, 1975).

\section{How does acaricide treatment compare to culling for controlling ticks and increasing grouse abundance?}

The model was used to predict the effect over time of treating deer with acaricide of efficacy $70 \%$ and culling $70 \%$ of the initial deer population for 2 initial (pre-management) deer densities $\left(10\right.$ and $20 \mathrm{~km}^{-2}$ ) (Fig. 4). For initial deer densities of $10 \mathrm{~km}^{-2}$ the tick density was predicted to decline dramatically and rapidly while the grouse density was predicted to reach carrying capacity $\left(240 \mathrm{~km}^{-2}\right)$ after 10 years for both acaricide treatment and culling. At original deer densities of $20 \mathrm{~km}^{-2}$ the model predicted that the tick 
(a) predicted grouse densities without treatment

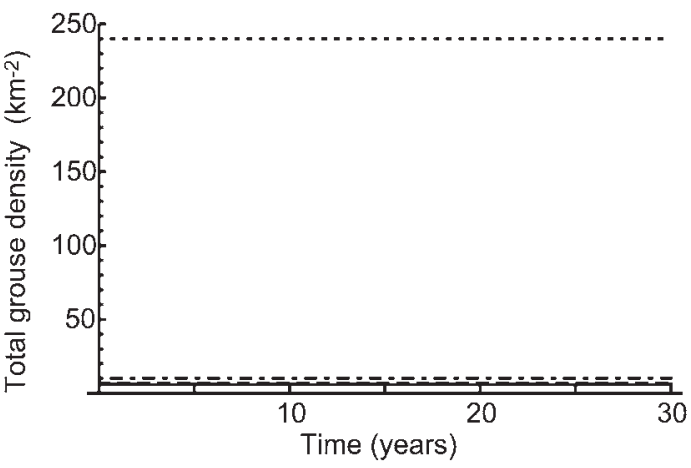

(b) predicted tick densities without treatment

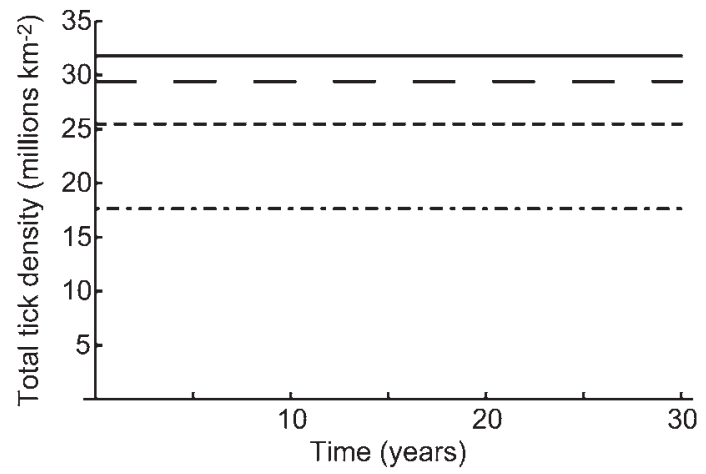

(c) predicted grouse densities with treatment

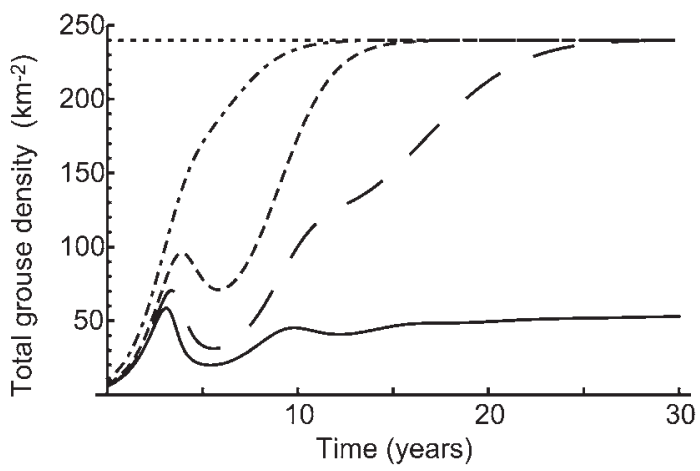

(d) predicted tick densities with treatment

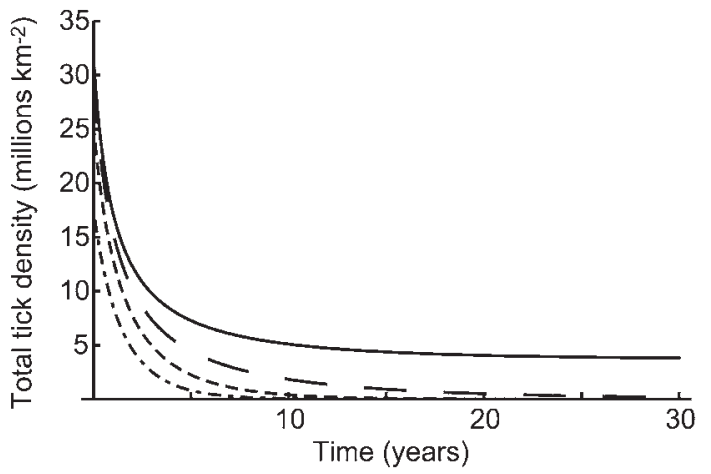

Fig. 1. The model predictions of total grouse and total tick densities over time for different deer densities with (a,b) and without (c,d) acaricide treatment. The deer densities $\left(\mathrm{km}^{-2}\right)$ are 5 (dotted), 10 (dot-dashed), 15 (short dashed), 20 (long dashed) and 25 (solid). Acaricide efficacy was set at 70\%.

density would decline less and more slowly for culling as compared to treating the deer, while the grouse density was predicted to eventually reach carrying capacity in the presence of treated deer, but not if deer were culled instead. Note that the model predicted that a higher initial deer density means that both culling and treatment are less effective than when there are fewer deer initially.

Could combining acaricide treatment with deer culling be the most effective management strategy for controlling LIV and increasing grouse abundance?

The model predicted that, at lower deer densities, the acaricide efficacy required to achieve grouse densities high enough for commercial hunting is lower (Fig. 3), and therefore more easily achievable in terms of practical management. For higher deer densities, higher acaricide efficacies were predicted to be necessary, and these may be difficult and/or expensive to attain in practice. One option, therefore, may be to first reduce the deer density by culling and then treat the remaining deer population with acaricide. For example, if a given acaricide treatment is thought to have an efficacy of $50 \%$ then the model predicts that the deer density may need to be reduced below $12 \mathrm{~km}^{-2}$ if grouse are to reach carrying capacity or a density high enough for commercial hunting (Fig. 3).
However, it should be noted that model predictions suggest that treating a deer population that has just been reduced by culling is not quite the same as treating a deer population that was already small. For example, treating 10 deer $\mathrm{km}^{-2}$ immediately after a cull from 20 deer $\mathrm{km}^{-2}$ is predicted to act more slowly on the tick and grouse populations than if there were only 10 deer $\mathrm{km}^{-2}$ initially, although ultimately the grouse are predicted to reach the same equilibrium density.

\section{DISCUSSION}

We used a modelling approach to investigate whether treating rather than culling a key parasite host can protect a valuable susceptible species from disease. In particular, using the red grouse-LIV system as our case study we asked (1) can acaricide-treated deer increase grouse abundance by reducing tick abundance, (2) what is the effect of acaricide efficacy on LIV persistence and grouse abundance, (3) how does culling compare to acaricide treatment for controlling ticks and increasing grouse abundance and 4) could combining acaricide treatment with deer culling be more effective?

The model predicted quantitative values, but we emphasize that actual values will vary as environmental conditions vary. Sensitivity analysis showed 


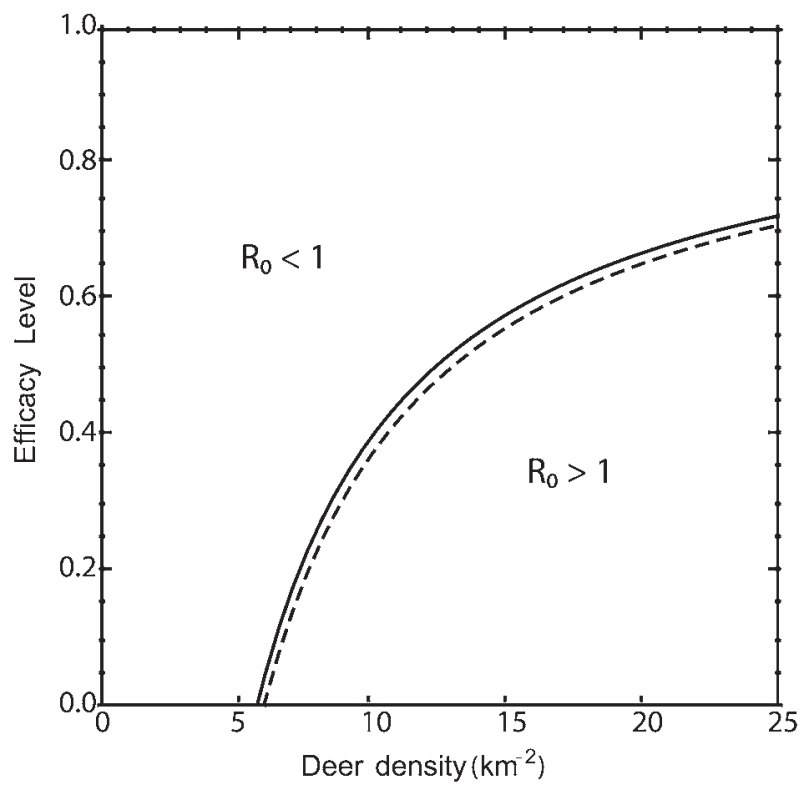

Fig. 2. The curve described by $R_{0}=1$ for different deer densities and acaricide efficacies. Above and to the left of the curve the virus dies out, below the curve the virus persists. The solid line denotes when the grouse population reaches carrying capacity $\left(240 \mathrm{~km}^{-2}\right)$. The dashed line denotes when the grouse population reaches an economically viable density for 'driven shooting' $\left(60 \mathrm{~km}^{-2}\right)$.

that the parameters to which the model was most sensitive were tick birth and death parameters and the biting rate of adult ticks on deer $\left(\beta_{5}\right)$, but the qualitative behaviour of the model was not altered by changing the parameter values by $10 \%$ in each direction and the quantitative results did not change greatly. However, there is still a great deal of variability in natural systems and so the values given by the model should be, at best, used as a rough guide only or used for the general patterns predicted.

(1) The model predicted that deer treated with acaricide improved grouse numbers by reducing tick numbers compared to the scenario of untreated deer populations. However, in the case of both treated and untreated deer populations, if deer density increased, so did tick density, causing a decline in grouse density (due to increased mortality from LIV). This is an expected outcome in the case of untreated deer, because more deer feed more ticks. However, this is less intuitive in the situation of deer treated with acaricide because, as the deer population increases, the number of ticks being killed by the acaricide will increase. However, because the efficacy was only $70 \%$, there is an increase in the number of ticks that survive as the deer population increases. For $70 \%$ efficacy, the increase in the numbers of ticks killed by acaricide is not enough to outweigh the increase in ticks fed by the growing deer population.

(2) The model predicted that the acaricide efficacy required to improve grouse density depended on deer density. For example, the model predicted that an acaricide efficacy of $80 \%$ allows grouse densities to become large enough for commercial hunting even in the presence of high deer densities (up to $25 \mathrm{~km}^{-2}$ ). However, if only intermediate acaricide efficacies (45-65\%) could be achieved, grouse will reach densities suitable for commercial hunting only if deer densities are lower $\left(12-18 \mathrm{~km}^{-2}\right)$. At very low deer densities the model predicted that no acaricide treatment is necessary because there are not enough deer to maintain a large enough tick population for LIV maintenance. This also suggests that deer densities need only be reduced rather than eradicated. However, we emphasize that these exact values of acaricide efficacy and deer density predicted by the model may not be accurate, and will certainly vary depending on local environmental variables such as climate and alternative tick hosts.

(3) Testing how acaricide treatment compared to culling deer the model predicted that, for fairly typical deer densities $\left(10 \mathrm{~km}^{-2}\right)$, culling $70 \%$ of the initial deer population had a very similar effect on ticks and grouse as treating deer with acaricide of $70 \%$ efficacy. However, for very high deer densities $\left(20 \mathrm{~km}^{-2}\right)$ acaricide treatment out-performed culling in terms of reducing tick densities and improving grouse densities. The model assumed that acaricide kills ticks that try to attach. In contrast, culling deer removes potential meals for ticks, but some of these ticks can instead feed on the remaining hosts.

We used $70 \%$ proportions and rates to test culling versus acaricide treatment to match the efficacies during trials using acaricide applicators on white-tailed deer in the USA (Pound et al. 2000; Solberg et al. 2003; Sonenshine et al. 1996). A 70\% cull of the initial deer population was necessary in the model to act as an equivalent comparison to the acaricide efficacy. However, this is an extremely high cull rate and in practice it might be difficult to achieve as well as raising ethical issues. As a general guide approximately $17 \%$ of a red deer population in Scotland might be culled per year in order to maintain a relatively constant deer density (Clutton-Brock and Albon, 1989) although cull rates vary between areas depending on the management objectives of the area. An initial cull of $70 \%$ of the initial population would require an enormous extra effort, and densitydependent effects (which were not included in the model) may mean that deer densities do not respond proportionately to the initial proportion culled.

(4) The model outputs above suggest that lower deer densities are beneficial in terms of achieving a successful effect on grouse populations. Therefore, reducing deer densities by culling before applying acaricide treatment to the remaining deer could theoretically be a successful management option. In practical terms, this dual management option might be preferable to only culling or only treating with acaricide, depending on the initial deer density and the needs of the land manager (e.g. how much income 
(a) predicted grouse densities

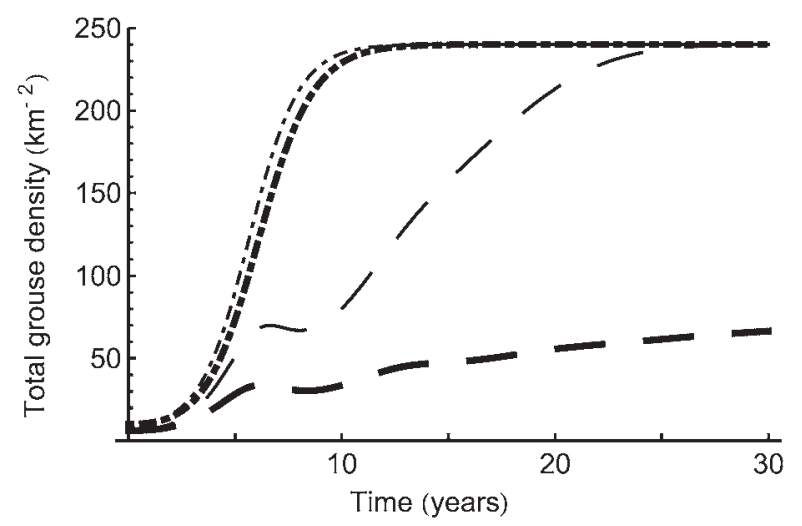

(b) predicted tick densities

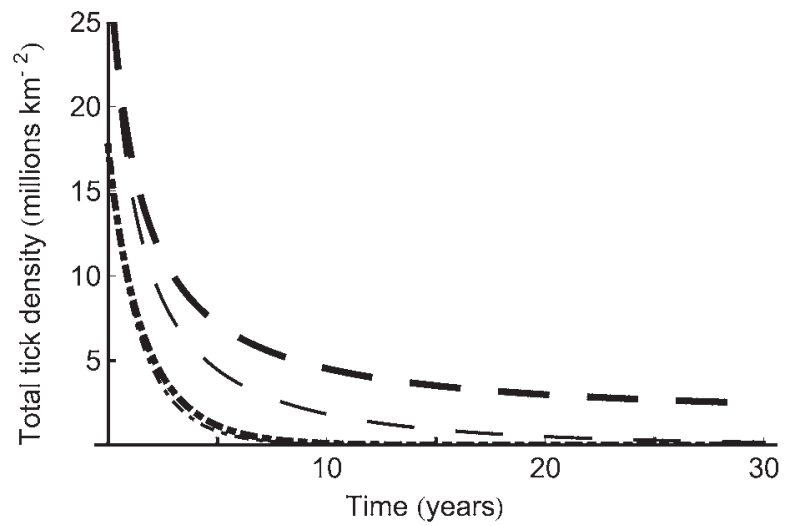

Fig. 3. The model predictions for (a) grouse and (b) tick densities for initial deer densities of $10 \mathrm{~km}^{-2}$ (dot-dashed) and $20 \mathrm{~km}^{-2}$ (long dashed). Treating deer with acaricide of $70 \%$ efficacy (thin lines) is compared with culling deer at a rate of $70 \%$ (thick lines).

is needed from maintaining enough deer for commercial hunting). Management decisions will also depend on practical, economic, ethical and legal issues, discussed below.

\section{Model limitations}

Whilst we are confident in the broad, qualitative, predictions of the model, (from our sensitivity analyses) models are simplifications of a system and need to make assumptions. The model did not incorporate space, so assumed a homogeneous environment where deer, grouse and ticks are equally mixed, yet landscapes are heterogeneous and host populations are not evenly distributed in space. This may affect the model outputs; for example, if deer do not occupy the same areas as the grouse, the effect of deer on ticks will have less of an effect on grouse than predicted. The model also assumed that deer, grouse and ticks are at a constant density throughout the year, even though offspring are produced and ticks become active in the spring. However, we are confident that this type of seasonality will not greatly change the patterns of the model predictions, since a seasonal model of LIV dynamics has been developed and outputs were similar to a non-season model (Porter, 2011). Seasonality will, however, be an important consideration for the practical timing of acaricide application in terms of practical management, which we discuss later.

Sheep and mountain hares, which can be alternative tick and LIV hosts on UK grouse moors, were not included in the model, so our model predictions will be much less accurate if applied to grouse moors where these hosts occur in large numbers. However, in some areas where commercial grouse hunting is deemed to be severely limited by LIV, the land managers are highly likely to be already removing sheep from the LIV transmission cycle by either removing them from the area or applying acaricides

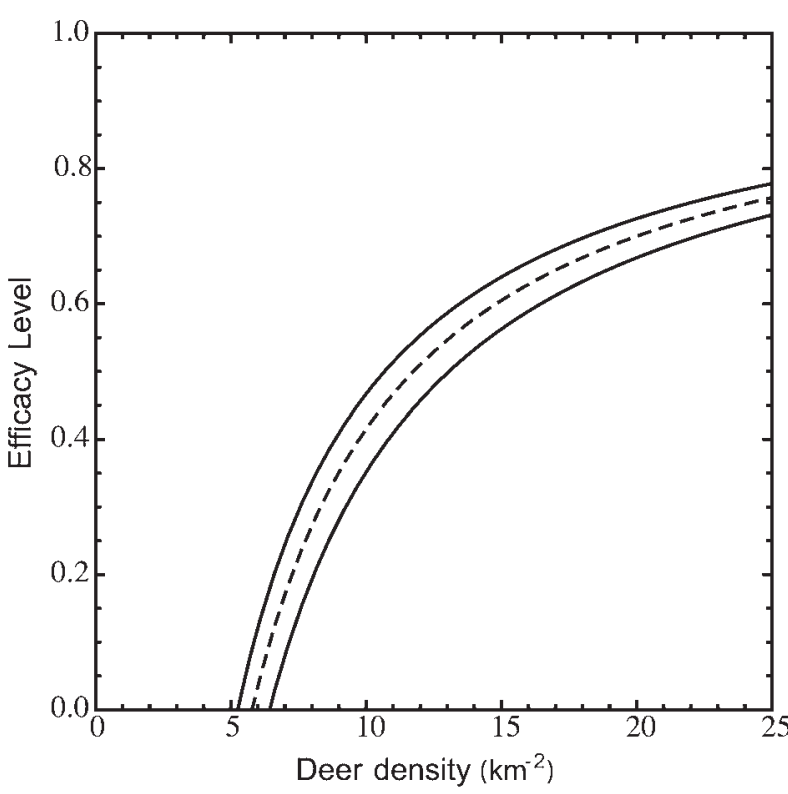

Fig. 4. The curve described by $R_{0}=1$ for different deer densities and acaricide efficacies to demonstrate the impact of using different tick biting rate values of ticks on deer $\left(\beta_{5}\right)$. The dotted line represents $R_{0}=1$ for the baseline parameter values given in Table 1 and the solid lines are when we use $\beta_{5} \pm 10 \%$.

and vaccinating them against LIV (see Laurenson et al. 2003; Porter et al. 2011). It is also likely that land managers will be already maintaining mountain hares at low densities (e.g. Laurenson et al. 2003). In addition, model evidence suggests that the role that mountain hares play in tick and LIV maintenance may not be as great as is the role of deer (Gilbert et al. 2001). Other hosts such as small mammals can also help feed immature ticks and were not in the model, although they tend to occur in relatively low numbers in Calluna-dominated heather moors (Gilbert et al. 2000) and predators such as foxes are usually controlled. However, we recognize that if alternative hosts were added to the model it is likely that an 
increase in acaricide efficacy would be required to counteract their presence. In addition, alternative hosts in the model would prevent the model from predicting unrealistic tick 'extinctions' when the deer population falls below a certain threshold density, since $I$. ricinus are generalist feeders that would be maintained by the presence of hosts such as hares, sheep, small mammals or wading birds.

\section{Practical management considerations}

The model predicted that treating deer with acaricide could, theoretically, be effective in reducing tick populations and increasing red grouse abundance if acaricide efficacy is high and deer density is medium or low. If this method is to be realistically considered for tick control, there are many issues that must be taken into account. First, the legalities for using the acaricide must be ascertained for the country in question, e.g. in the UK commercial acaricides are licensed for domestic animals, so if this control method was to be considered for wild deer in the UK licencing procedures would be needed. It would also be important to identify whether ticks and LIV really are limiting the particular grouse population in question, rather than other factors such as habitat quality, predators or gastro-intestinal nematodes.

Assuming ticks and LIV are limiting the grouse population in question, how practical and realistic might it be to achieve the acaricide efficacies required on wild deer to produce a dramatic reduction in tick abundance? During a series of studies conducted in the USA white-tailed deer were attracted to patented '4-poster' feeding stations where they rubbed their heads against rollers impregnated with acaricide, and this could successfully reduce the abundance of questing I. scapularis ticks by $70 \%$, averaged over all trialled areas in 5 states (summarized by Pound et al. 2009). While our model predicted a similar successful reduction in ticks with high herd-level acaricide efficacies, we need to examine whether such high efficacies might be achievable for wild deer on UK grouse moors. The most abundant and widely distributed deer species in the UK that are regularly found in the same habitat as red grouse are red deer and European roe deer Capreolus capreolus. These species have important differences and the practicalities of treating them are likely to differ. There are several potential reasons why high efficacies might be difficult to achieve for wild red deer on UK grouse moors. (i) Carroll et al. (2009) suggested that a lower proportion of white-tailed deer individuals would gain access to applicator devices in larger herds (>30 individuals), due to subordinates being excluded by more dominant individuals. Red deer typically form large herds and so a potential issue could be that a smaller proportion of individuals may get adequately treated with acaricide than that reported for white-tailed deer (mean 76\% (range 12-90\%); Pound et al. 2009). (ii) All the trials in the USA reported less usage of the applicator devices when alternative food (such as oak mast) was very abundant (summarized by Pound et al. 2009). On UK grouse moors, natural food for red deer is most abundant during the summer, roughly coinciding with the main I. ricinus activity period. Therefore, red deer may be most likely to use applicator devices in the winter when there are fewer questing ticks for the acaricide to kill. In addition, on UK grouse moors, red deer tend to move up to higher altitudes in the summer, which will greatly increase the effort and resources in maintaining feeding stations during the tick questing season. (iii) White-tailed deer carry the vast majority of the I. scapularis ticks on the head, ears, neck and chest (Schmidtmann et al. 1998) and so acaricide applied from a '4-poster' type station can reach these areas of the animal. We have count data of I. ricinus on 2 whole red deer carcasses from Scotland: only $42 \%$ of $I$. ricinus were on the head, ears, neck and chest, with most ticks attaching to the inguinal areas at the back of the animal (Gilbert, unpublished data). Stafford et al. (2009) reported that white-tailed deer that had been treated using a '4-poster' carried $76 \%$ of I. scapularis ticks on the back leg/groin areas, exhibiting successful control of ticks on the head and front of the animals, and suggesting that the acaricide does not always successfully control ticks on body parts furthest away from the point of application. Empirical trials are needed to explore these concerns about practicalities with red deer.

It is possible that treating European roe deer with acaricide using feeding stations might be more appropriate than treating red deer, since roe deer do not form large herds where subordinate individuals may be excluded from application devices and, in addition, the majority of ticks occur on the head and neck of roe deer (Kiffner et al. 2011). Although some roe deer frequent upland habitats such as grouse moors, roe deer in the $\mathrm{UK}$ tend to be more abundant in woodlands (which is not habitat to red grouse) so targeting roe deer might not necessarily successfully protect grouse from LIV, except in places where roe deer frequent the grouse moor and where there are few red deer. Treating roe deer could perhaps have the potential to reduce ticks and Lyme borreliosis risk for humans, in a similar way demonstrated in the USA with white-tailed deer (Hoen et al. 2009), and this may be worth investigation.

The application of acaricide to wildlife has generic issues, such as the potential for acquired resistance to the acaricides by the ticks, and the possible necessity for withdrawal periods between acaricide application and hunting the host species if they are used for human consumption.

We have used a mathematical model to theoretically predict the effectiveness of treating rather than culling one host species to benefit a second 
susceptible species by reducing the effect of parasitemediated apparent competition between the two host species. Our models predicted that treating deer with acaricide may help protect red grouse from ticks and LIV under certain circumstances (such as high acaricide efficacy and low deer densities). However, it would be essential to run small- and large-scale trials before consideration as a practical management tool, to investigate empirically the potential practical difficulties that we discussed with treating enough individuals of both roe and red deer. Other issues must also be considered such as the legality of using acaricides on wildlife, as well as practical, economic, health and welfare issues. The methods described here are directly applicable to other tick-borne diseases, such as TBEv and Lyme borreliosis. Our approach could also be useful for exploring alternative methods of disease control in other host-pathogen systems, such as comparing the effectiveness of culling versus vaccinating badgers against $\mathrm{bTB}$ for controlling bTB in cattle, or culling grey squirrels versus using immuno-contraceptives (or vaccination against squirrel poxvirus if a vaccine is developed) for protecting red squirrel populations.

\section{ACKNOWLEDGEMENTS}

We thank Justin Irvine, Pete Goddard and two anonymous referees for comments on the manuscript.

\section{FINANCIAL SUPPORT}

R.P. was funded by a Natural Environment Research Council studentship (NE/H527316/1) and the Macaulay Development Trust, awarded to L.G. and R.N. L.G. was funded by the Scottish Government Rural Environment Research and Analysis Directorate.

\section{REFERENCES}

Anderson, R. M. and May, R. M. (1981). The population-dynamics of micro-parasites and their invertebrate hosts. Philosophical Transactions of the Royal Society of London, B 291, 451-524.

Brei, B., Brownstein, J.S., George, J. E., Pound, J. M., Miller, J. A., Daniels, T. J., Falco, R.C., Stafford, K. C., Schulze, T. L., Mather, T. N., Carroll, J. F. and Fish, D. (2009). Evaluation of the United States Department of Agriculture Northeast Area-Wide Tick Control Project by meta-analysis. Vector-Borne and Zoonotic Diseases 9 , 423-430.

Carroll, J. F., Hill, D. E., Allen, P. C., Young, K. W., Miramontes, E., Kramer, M., Pound, J. M., Miller, J. A., and George, J. E. (2009). The impact of '4-poster' deer self-treatment devices at three locations in Maryland. Vector-Borne and Zoonotic Diseases 9, 407-416.

Clutton-Brock, T., and Albon, S. (1989). Red Deer in the Highlands. BSP Professional Books.

Gaunt, M. W. (1997). The epidemiology of louping ill virus and lavivirus evolution. Ph.D. thesis, University of Oxford, Oxford, UK.

Gilbert, L., Jones, L. D., Hudson, P. J., Gould, E. A. and Reid, H. W. (2000). Role of small mammals in the persistence of Louping-ill virus: field survey and tick co-feeding studies. Medical and Veterinary Entomology 14, $277-282$.

Gilbert, L., Norman, R., Laurenson, M. K., Reid, H. W. and Hudson, P. J. (2001). Disease persistence and apparent competition in a three-host community: an empirical and analytical study of large-scale, wild populations. Fournal of Animal Ecology 70, 1053-1061.

Gray, J.S. (1998). The ecology of ticks transmitting Lyme borreliosis. Experimental and Applied Acarology 22, 249-258.
Hartemink, N. A., Randolph, S.E., Davis, S. A. and Heesterbeek, J. A. P. (2008). The basic reproduction number for complex disease systems: Defining R0 for tick-borne infections. American Naturalist 171, 743-754

Hoen, A. G., Rollend, L. G., Papero, M. A., Carroll, J. F., Daniels, T. J., Mather, T.N., Schulze, T.L., Stafford III, K. C. and Fish, D. (2009). Effects of tick control of acaricide self-treatment of white-tailed deer onhost-seeking tick infection prevalence and entomologic risk for Ixodes scapularis-borne pathogens. Vector-Borne and Zoonotic Diseases 9, 431-438.

Holt, R. D. (1977). Predation, apparent competition, and the structure of prey communities. Theoretical Population Biology 12, 197-229.

Hudson, P. J. (1992). Grouse in Space and Time. Game Conservancy Trust, Fordingbridge, UK.

Jones, L. D., Gaunt, M., Hailes, R. S., Laurenson, K., Hudson, P. J., Reid, H., Henbest, P. and Gould, E. A. (1997). Transmission of louping ill virus between infected and uninfected ticks co-feeding on mountain hares. Medical and Veterinary Entomology 11, 172-176.

Kirby, A. D., Smith, A. A., Benton, T. G. and Hudson, P. J. (2004). Rising burden of immature sheep ticks Ixodes ricinus on red grouse Lagopus lagopus scoticus chicks in the Scottish uplands. Medical and Veterinary Entomology 18, 67-70.

Kulasekera, V. L., Kramer, L., Nasci, R.S., Mostashari, F., Cherry, B., Trock, S. C., Glaser, C. and Miller, J. R. (2001). West Nile virus infection in mosquitoes, birds, horses, and humans, Staten Island, New York, 2000. Emerging Infectious Diseases 7, 722-725.

Kiffner, C., Lödige, C., Alings, M., Vor, T. and Rühe, F. (2011). Attachment site selection of ticks on roe deer, Capreolus capreolus. Experimental and Applied Acarology 53, 79-94.

Laurenson, M.K., Norman, R. A., Gilbert, L., Reid, H.W. and Hudson, P. J. (2003). Identifying disease reservoirs in complex systems: mountain hares as reservoirs of ticks and louping-ill virus, pathogens of red grouse. Fournal of Animal Ecology 72, 177-185.

Newborn, D. and Baines, D. (2012). Enhanced control of sheep ticks in upland sheep flocks: repercussions for red grouse co-hosts. Medical and Veterinary Entomology 26, 63-69.

Porter, R., Norman, R. and Gilbert, L. (2011). Controlling tick-borne diseases through domestic animal management: a theoretical approach. Theoretical Ecology 4, 321-339.

Porter, R. (2011). Mathematical models of a tick borne disease in a British gamebird with potential management strategies. Ph.D. thesis, University of Stirling, Scotland, UK.

Pound, J.M., Miller, J. A., George, J. E., Fish, D., Carroll, J. F., Schulze, T. L., Daniels, T. J., Falco, R.C., Stafford III, K. C. and Mather, T. N. (2009). The United States Department of Agriculture's Northeast Area-Wide Tick Control Project: summary and conclusions. Vector-Borne and Zoonotic Diseases 9, 439-448.

Pound, J. M., Miller, J. A., George, J. E. and Lemeilleur, C. A. (2000). The "4-Poster" passive topical treatment device to apply acaricide for controlling ticks feeding on white-tailed deer. Fournal of Medical Entomology 37, 588-594.

Randolph, S. E. (2004). Evidence that climate change has caused 'emergence' of tick-borne diseases in Europe? International fournal of Medical Microbiology 293 S37, 5-15.

Reid, H. W. (1975). Experimental infection of red grouse with Louping-Ill virus flavivirus group. 1 Viremia and antibody-response. Fournal of Comparative Pathology 85, 223-229.

Ruiz-Fons, F. and Gilbert, L. (2010). The role of deer as vehicles to move ticks, Ixodes ricinus, between contrasting habitats. International fournal for Parasitology 40, 1013-1020.

Rushton, S. P., Lurz, P. W. W. and Gurnell, J. (2000). Modelling the spatial dynamics of parapoxvirus disease in red and grey squirrels: a possible cause of the decline in the red squirrel in the United Kingdom? Yournal of Applied Ecology 37, 1-18.

Schmidtmann, E. T., Carroll, J.F. and Watson, D.W. (1998). Attachment-site patterns of adult black-legged ticks on white-tailed deer and horses. Fournal of Medical Entomology 35, 59-63.

Solberg, V. B., Miller, J. A., Hadfield, T., Burge, R., Schech, J. M. and Pound, J. M. (2003). Control of Ixodes scapularis Acari: Ixodidae. with topical self-application of permethrin by white-tailed deer inhabiting NASA, Beltsville, Maryland. Fournal of Vector Ecology 28, 117-134. Sonenshine, D., Allen, S. A., Noval, R. A. and Burridge, M. J. (1996). A self-medicating applicator for control of ticks on deer. Medical and Veterinary Entomology 10, 149-154.

Stafford, K. C. 3rd, Denicola, A. J. and Kilpatrick, H. J. (2003). Reduced abundance of Ixodes scapularis (Acari: Ixodidae) and the tick parasitoid Ixodiphagus hookeri (Hymenoptera: Encyrtidae) with reduction of white-tailed deer. Fournal of Medical Entomology 40, 642-652. 
Stafford, K. C., III, Denicola, A. J., Pound, J. M., Miller, J. A. and George, J. E. (2009). Topical treatment of white-tailed deer with an acaricide for the control of Ixodes scapularis Acari: Ixodidae. in a Connecticut Lyme Borreliosis hyperendemic community. Vector-Borne and Zoonotic Diseases 9, 371-379.
Tompkins, D., Sainsbury, A.W., Nettleton, P., Buxton, D. and Gurnell, J. (2002). Parapoxvirus causes a deleterious disease of red squirrels associated with UK population declines. Proceedings of the Royal Society of London, B 269, 529-533. 